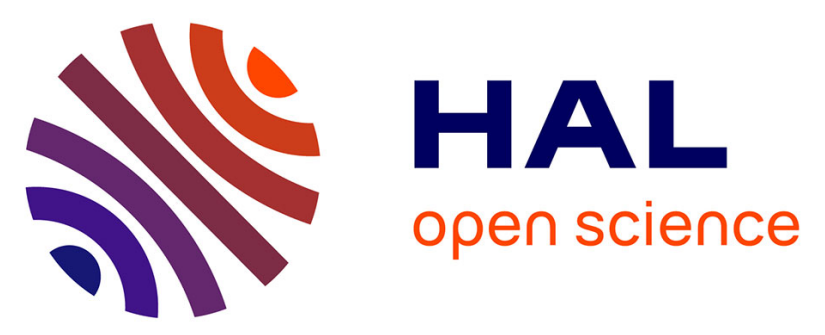

\title{
A Joint Multiplexing and Resource Allocation Algorithm for Asynchronous Underlay D2D Communications
}

\author{
Mylene Pischella, Rostom Zakaria, Didier Le Ruyet
}

\section{- To cite this version:}

Mylene Pischella, Rostom Zakaria, Didier Le Ruyet. A Joint Multiplexing and Resource Allocation Algorithm for Asynchronous Underlay D2D Communications. 2018 IEEE 87th Vehicular Technology Conference (VTC Spring), Jun 2018, Porto, Portugal. 10.1109/VTCSpring.2018.8417570 . hal02446291

\section{HAL Id: hal-02446291 https://hal-cnam.archives-ouvertes.fr/hal-02446291}

Submitted on 19 Mar 2020

HAL is a multi-disciplinary open access archive for the deposit and dissemination of scientific research documents, whether they are published or not. The documents may come from teaching and research institutions in France or abroad, or from public or private research centers.
L'archive ouverte pluridisciplinaire HAL, est destinée au dépôt et à la diffusion de documents scientifiques de niveau recherche, publiés ou non, émanant des établissements d'enseignement et de recherche français ou étrangers, des laboratoires publics ou privés. 


\title{
A joint multiplexing and resource allocation algorithm for asynchronous underlay D2D communications
}

\author{
Mylene Pischella, Rostom Zakaria and Didier Le Ruyet \\ CNAM CEDRIC/LAETITIA \\ 292 rue Saint-Martin - 75141 Paris Cedex 03, France \\ Email: mylene.pischella@cnam.fr; rostom.zakaria@cnam.fr; didier.le_ruyet@cnam.fr;
}

\begin{abstract}
This paper studies the joint multiplexing, ResourceBlock and power allocation problem in underlay Device-to-Device (D2D) communications. The interfering signals are assumed fully asynchronous at the device receivers and at the Base Station. Inter-Channel Interference (ICI) then generates major impairment on the expected rate gain if Orthogonal Frequency Division Multiplex (OFDM) multi-carrier modulation is used, contrary to new multi-carrier modulations such as Filter Bank Multi-Carrier (FBMC) or Fast Fourier Transform (FFT)-FBMC. The proposed centralized algorithm provides a lower bound to the maximum sum rate over D2D pairs, taking into account ICI. It increases the sum data rate compared to a Frequency Division Multiple Access (FDMA) technique and compared to two other algorithms allowing multiplexing and applying power control.
\end{abstract}

Index Terms: Multiplexing, resource allocation, Device-toDevice, multi-carrier modulations.

\section{INTRODUCTION}

Fifth Generation (5G) networks will allow direct transmissions between devices with minimum control requirements from the Base Station (BS). High data rate increases can be expected with Device-to-Device (D2D) communications if efficient frequency reuse techniques are applied within the cell [1]-[3]. In D2D underlay communications, D2D pairs may reuse the same Resource Blocks (RB) as cellular users, provided that the interference that they generate at the BS remains under a given threshold.

Most studies on 5G networks have assumed fully synchronized transmissions. However, this assumption is quite strong since each D2D receiver is only perfectly synchronized with its own transmitter, and propagation as well as multi-path delays between interfering D2D transmitters and a specific D2D receiver may have a large distribution. Asynchronicity generates Inter-Channel Interference (ICI), that may particularly degrade data rates when the multi-carrier modulation, like Orthogonal Frequency Division Multiplex (OFDM), is not well-localized in the frequency domain. Many new multi-carrier modulations have recently been studied for $5 \mathrm{G}$ and Beyond $5 \mathrm{G}$ networks. Among them, Filter Bank Multi-Carrier (FBMC) and Fast Fourier Transform (FFT)-FBMC are particularly well localized in the frequency domain [4].

In this paper, we consider the sum rate maximization problem in asynchronous D2D communications, with a maximum interference constraint per subcarrier at the BS. The D2D network is thus underlaid in the cellular network. The system model to take into account asynchronicity was determined in our previous letter [5], where we also proposed a distributed power allocation algorithm but we did not optimize RB allocation. In this paper, on the contrary, we consider the whole resource allocation problem and obtain a joint centralized algorithm determining the best D2D multiplexing, RB and power allocation. Most resource allocation algorithms for D2D communications separate RB allocation from power allocation and assume full synchronicity (see references in [6]). Joint RB and power allocation has only been studied in the literature with iterative processing: $\mathrm{RB}$ and power allocation steps are separated, but they are iterated in order to achieve better performance [7], [8]. The algorithm proposed in this paper goes further, as it solves RB and power allocation altogether by assuming that full frequency reuse is allowed, and letting the outcome of power allocation decide of RB allocation. Moreover, this algorithm takes into account potential asynchronicity.

The paper is organized as follows: section II presents the system model. The proposed joint multiplexing and resource allocation algorithm is detailed in section III. It is then compared with three reference algorithms in asynchronous transmissions with OFDM, FBMC and FFT-FBMC in section $\mathrm{IV}$, and section $\mathrm{V}$ concludes the paper.

\section{SYSTEM MODEL}

We consider $K$ D2D pairs and $C$ cellular users operating in the uplink of an isolated cell. All transmitters and receivers are equipped with only one antenna. D2D pairs are underlaid a in cellular multi-carrier network with $N$ RB composed of $M$ adjacent subcarriers. $L=M \times N$ is the total number of subcarriers. Cellular users are already allocated and may interfere D2D receivers. The corresponding interference is included in the interference plus noise term of receiver $k$ and subcarrier $l, n_{k}^{l}$, which also contains thermal noise. Resource allocation optimization only concerns D2D transmissions.

The system model takes into account the ICI generated by asynchronous transmissions and is determined so that power is allocated per RB, even though ICI is defined per subcarrier. The complete system model for asynchronous D2D transmissions with per RB power allocation has been determined in [5]. In this paper, we build on this system model but do not describe it in details due to lack of space.

The ICI weights are modelled as a vector $\mathbf{V}$ of size $L$ that spreads over $v$ subcarriers, where $V_{\left|l-l^{\prime}\right|}=0$ if $\left|l-l^{\prime}\right|>v$. Its spread and amplitude depend on the multi-carrier modulation. For instance, we provide the values of $\mathbf{V}$ with LTE parameters, when the multi-carrier symbol durations is $T=66.6 \mu \mathrm{s}$, the cyclic prefix $(\mathrm{CP})$ duration is $\Delta=4.69 \mu \mathrm{s}$ and the timing 
offset is uniformly distributed in $[0 ; T+\Delta]$ for OFDM and in $[0 ; T]$ for FBMC and FFT-FBMC. Only weights exceeding $10^{-3}$ are considered. The frequency spread $v$ is equal to 9 with OFDM and 1 with FBMC if the PHYDYAS filter is used [9], and with FFT-FBMC when the precoding FFT size is 64 . The $v+1$ non-zero elements of vector $\mathbf{V}$ are equal to:

$$
\begin{aligned}
\mathbf{V}_{\text {OFDM }}= & {\left[6.89 \times 10^{-1}, 9.47 \times 10^{-2}, 2.37 \times 10^{-2}\right.} \\
& 1.05 \times 10^{-2}, 5.9 \times 10^{-3}, 3.8 \times 10^{-3}, \\
& \left.2.6 \times 10^{-3}, 1.9 \times 10^{-3}, 1.5 \times 10^{-3}, 1.12 \times 10^{-3}\right] \\
\mathbf{V}_{\text {FBMC }}= & {\left[8.23 \times 10^{-1}, 8.81 \times 10^{-2}\right] } \\
\mathbf{V}_{\text {FFT-FBMC }}= & {\left[9.68 \times 10^{-1}, 6.9 \times 10^{-3}\right] }
\end{aligned}
$$

The reference vector is $\mathbf{V}_{\mathrm{PS}}=$ [1] for Perfectly Synchronized (PS) transmission, which represents a theoretical upper-bound with $\mathrm{CP} \Delta=0$. In FFT-FBMC, the CP is also set to 0 , since in Single Input, Single Output (SISO) transmissions with a 64-point FFT, good performance is achieved even without any CP (see Table III in [4]).

Including $\mathbf{V}$ and the per-RB power allocation constraint in the system model, we obtain the following expression for the data rate of D2D receiver $k$ in RB $r$, with $\log (x)=\log _{2}(x)$ :

$$
D_{k}^{r}(\mathbf{p})=\sum_{l \in \mathbb{R}_{r}} \alpha_{k} \log \left(1+\frac{F_{r k}^{l k} P_{k}^{r}}{n_{k}^{l}+I_{k}^{l}}\right)
$$

where:

- $\mathbf{p}$ is the vector of all transmitted D2D powers in all RB, with $P_{k}^{r}=\mathbf{p}(r+k N)$ the transmitted power of D2D user $k$ in RB $r$, assumed equal in all $M$ subcarriers of RB $r$,

- $F_{r k}^{l k}$ is the direct channel gain between transmitter $k$ and its receiver in subcarrier $l$,

- $F_{r j}^{l k}$ represents the interference coefficient from transmitter $j$ active in RB $r$ to receiver $k$ in subcarrier $l$ (with $k \neq j$ ), including the channel gain with squared modulus of fading, shadowing and path loss, and ICI weights,

- $I_{k}^{l}=\sum_{\substack{j=0 \\ j \neq k}}^{K-1} \sum_{r \in \mathbb{B}_{j}} F_{r j}^{l k} P_{j}^{r}$ is the interference received in subcarrier $l$ by receiver $k$,

- $\mathbb{R}_{r}$ is the index set of the subcarriers in the RB $r$.

\section{JOINT MULTIPLEXING AND RESOURCE ALLOCATION}

In this section, we propose a centralized algorithm to jointly allocates RB and power to D2D pairs. This algorithm determines the power values of each D2D transmitter. Then $\mathrm{RB} r$ is allocated to D2D pair $k$ if $P_{k}^{r}>0$ at the end of the resource allocation algorithm. The set of D2D pairs that are multiplexed on RB $r$ is the set of pairs $k$ such that $P_{k}^{r}>0$. Consequently, and contrary to most previous papers on D2D resource allocation, a single joint algorithm obtains D2D multiplexing, RB and power allocation.

The optimization problem aims at maximizing the sum rate of D2D pairs, given that the cellular users are already allocated and generate fixed interference at D2D receivers. Each D2D transmitter has a sum power limit equal to $P_{\max }$, and the interference per subcarrier at the BS must be less than $I_{0}$. $A_{k r}^{l}$ is the interference coefficient at the BS in subcarrier $l$ from transmitter $k$ and $\mathrm{RB} r$, that includes the channel gain from $\mathrm{D} 2 \mathrm{D}$ transmitter $k$ in $\mathrm{RB} r$ to the $\mathrm{BS}$ in subcarrier $l$, and ICI weights.
The optimization problem is the following:

$$
\begin{aligned}
& \max _{\mathbf{p} \geq 0} \sum_{k=0}^{K-1} \sum_{r=0}^{N-1} D_{k}^{r}(\mathbf{p}) \\
& \text { s.t. } M \sum_{r=0}^{N-1} P_{k}^{r} \leq P_{\max } \forall k \in\{0, \ldots, K-1\} \\
& \text { s.t. } \sum_{k=0}^{K-1} \sum_{r=0}^{N-1} A_{k r}^{l} P_{k}^{r} \leq I_{0} \forall l \in\{0, \ldots, L-1\}
\end{aligned}
$$

We can notice that the only optimization variables are the D2D transmit power values $p$. We indeed solve the joint resource allocation by allowing any D2D pair to be active in any RB. Then the power allocation is responsible of determining the RB allocation. This strategy provides the best $\mathrm{RB}$ allocation since it does not include any constraint on RB allocation within the optimization problem, that would lead to sub-optimal solutions. Moreover, we do not insert any perRB power constraint to let D2D transmissions be as effective as possible. Since the final transmit power of D2D are low because of the BS interference constraint, the final D2D power per RB most likely still fits within a power mask.

Problem (5) is not convex because the objective function is not concave. A series of approximation by convex optimization problems [10] can be used in order to reach a lower bound to problem (5). The objective function $D_{k}^{r}(\mathbf{p})$ is written as:

$$
D_{k}^{r}(\mathbf{p})=f_{k}^{r}(\mathbf{p})-g_{k}^{r}(\mathbf{p})
$$

where

$$
f_{k}^{r}(\mathbf{p})=\sum_{l \in \mathbb{R}_{r}} \log \left(n_{k}^{l}+\sum_{\substack{j=0 \\ j \neq k}}^{K-1} \sum_{r^{\prime}=0}^{N-1} F_{r^{\prime} j}^{l k} P_{j}^{r^{\prime}}+F_{r k}^{l k} P_{k}^{r}\right)
$$

and

$$
g_{k}^{r}(\mathbf{p})=\sum_{l \in \mathbb{R}_{r}} \log \left(n_{k}^{l}+\sum_{\substack{j=0 \\ j \neq k}}^{K-1} \sum_{r^{\prime}=0}^{N-1} F_{r^{\prime} j}^{l k} P_{j}^{r^{\prime}}\right)
$$

$f_{k}^{r}$ and $g_{k}^{r}$ are concave in $\mathbf{p}$ but $D_{k}^{r}$ is not. Consequently, the first order Taylor approximation on $g_{k}^{r}$ can be used in order to obtain a concave lower bound to $D_{k}^{r}$.

This approximation is included in an iterative algorithm that starts from a feasible point of (5) and then updates $p$ at iteration $t+1$ by solving a convex optimization problem with the first Taylor approximation of $g_{k}^{r}$ around the feasible point $\mathbf{p}$ obtained at iteration $t$.

Let $\overline{\mathbf{p}}_{t}$ be a feasible power vector obtained at iteration $t$. Then at iteration $t+1$, the first order Taylor approximation of $g_{k}^{r}$ around $\overline{\mathbf{p}}_{t}$ such that $\overline{P_{j, t}^{r}}=\overline{\mathbf{p}}_{t}(r+j N)$ is defined as follows:

$$
\widehat{g}_{k}^{r}\left(\mathbf{p}, \overline{\mathbf{p}_{t}}\right)=g_{k}^{r}\left(\overline{\mathbf{p}_{t}}\right)+\nabla g_{k}^{r}(\overline{\mathbf{p}})^{T}\left(\mathbf{p}-\overline{\mathbf{p}_{t}}\right)
$$

Where $\nabla g_{k}^{r}(\overline{\mathbf{p}})$ is the gradient of $g_{k}^{r}$ at vector $\overline{\mathbf{p}}$. Let us define:

$$
\overline{a_{k, t}^{r}}=g_{k}^{r}\left(\overline{\mathbf{p}_{t}}\right)
$$

and 


$$
\overline{b_{r_{0}^{\prime} j_{0}, t}^{r k}}=\frac{1}{\log (2)} \sum_{l \in \mathbb{R}_{r}}\left(\frac{F_{r_{0}^{\prime} j_{0}}^{l k}}{n_{k}^{l}+\sum_{\substack{j=0 \\ j \neq k}}^{K-1} \sum_{r^{\prime}=0}^{N-1} F_{r^{\prime} j}^{l k} \overline{P_{j, t}^{r^{\prime}}}}\right)
$$

Then eq. (9) can be written as:

$$
\widehat{g}_{k}^{r}\left(\mathbf{p}, \overline{\mathbf{p}_{t}}\right)=\overline{a_{k, t}^{r}}+\sum_{\substack{j_{0}=0 \\ j_{0} \neq k}}^{K-1} \sum_{r_{0}^{\prime}=0}^{N-1} \overline{b_{r_{0}^{\prime} j_{0}, t}^{r k}}\left(\mathbf{p}-\overline{\mathbf{p}_{t}}\right)
$$

where the double sum on $j_{0}$ and $r_{0}^{\prime}$ is due to the scalar product between $\nabla g_{k}^{r}(\overline{\mathbf{p}})$ and vector $\left(\mathbf{p}-\overline{\mathbf{p}_{t}}\right)$ in (9).

The first order Taylor approximation is an upper bound to $g_{k}^{r}$ because $g_{k}^{r}$ is concave. Consequently, a lower bound to $D_{k}^{r}$ is given by:

$$
\widehat{D}_{k, t}^{r}\left(\mathbf{p}, \overline{\mathbf{p}_{t}}\right)=f_{k}^{r}(\mathbf{p})-\widehat{g}_{k, t}^{r}\left(\mathbf{p}, \overline{\mathbf{p}_{t}}\right)
$$

and $\widehat{D}_{k, t}^{r}\left(\mathbf{p}, \overline{\mathbf{p}_{t}}\right)$ is a concave function in $\mathbf{p}$ since it is the sum of $\log$ and linear functions:

$$
\begin{aligned}
\widehat{D}_{k, t}^{r}\left(\mathbf{p}, \overline{\mathbf{p}_{t}}\right) & =\sum_{l \in \mathbb{R}_{r}} \log \left(n_{k}^{l}+\sum_{\substack{j=0 \\
j \neq k}}^{K-1} \sum_{r^{\prime}=0}^{N-1} F_{r^{\prime} j}^{l k} P_{j}^{r^{\prime}}+F_{r k}^{l k} P_{k}^{r}\right) \\
& -\overline{a_{k, t}^{r}}-\sum_{\substack{j_{0}=0 \\
j_{0} \neq k}}^{K-1} \sum_{r_{0}^{\prime}=0}^{N-1} \overline{b_{r_{0}^{\prime} j_{0}, t}^{r k}}\left(P_{j_{0}}^{r_{0}^{\prime}}-\overline{P_{j_{0}, t}^{r^{\prime}}}\right)
\end{aligned}
$$

Finally, the optimization problem at iteration $t+1$ is:

$$
\begin{aligned}
& \max _{\mathbf{p} \geq 0} \sum_{k=0}^{K-1} \sum_{r=0}^{N-1} \widehat{D}_{k, t}^{r}\left(\mathbf{p}, \overline{\mathbf{p}_{t}}\right) \\
& \text { s.t. } M \sum_{r=0}^{N-1} P_{k}^{r} \leq P_{\max } \forall k \in\{0, \ldots, K-1\} \\
& \text { s.t. } \sum_{k=0}^{K-1} \sum_{r=0}^{N-1} A_{k r}^{l} P_{k}^{r} \leq I_{0} \forall l \in\{0, \ldots, L-1\}
\end{aligned}
$$

This problem is convex in $\mathbf{p}$ and can be solved with standard optimization tools such as CVX [11]. The global algorithm is summarized in Algorithm 1.

Let $D_{\text {sum }, t}\left(\mathbf{p}, \overline{\mathbf{p}_{t}}\right)=\sum_{k=0}^{K-1} \sum_{r=0}^{N-1} \widehat{D}_{k, t}^{r}\left(\mathbf{p}, \overline{\mathbf{p}_{t}}\right)$ be the objective function at iteration $t$ and $\mathbf{p}_{t}^{*}$ the optimum power vector solving problem (13). Since this problem was solved starting from vector $\overline{\mathbf{p}_{t}}=\mathbf{p}_{t-1}^{*}$, the value of the objective function at iteration $t$ is necessarily larger than that at iteration $t-1$, $D_{\text {sum }, t}\left(\mathbf{p}, \overline{\mathbf{p}_{t}}\right) \geq D_{\text {sum }, t-1}\left(\mathbf{p}, \overline{\mathbf{p}_{t-1}}\right)$. The sequence of sum rate values $\left\{D_{\text {sum }, t}\left(\mathbf{p}, \overline{\mathbf{p}_{t}}\right)\right\}_{0<t<\infty}$ is a monotonically increasing sequence of feasible solutions that is upper-bounded by the optimum of the initial problem (5). Consequently, it converges to a feasible solution of (5). Its convergence is numerically evaluated in section IV.

The computational complexity of solving problem (13) is in $\mathcal{O}\left((K N)^{1.5}(K+L)^{2} \ln (1 / \delta)\right)$, where $\delta$ is the accuracy of the solver, that uses interior-point methods [12]. For instance with CVX, the accuracy is $1.49 \times 10^{-8}$ and $\ln (1 / \delta) \approx 18$. Computations of $\overline{a_{k, t}^{r}}$ and $\overline{b_{r_{0}^{\prime} j_{0}, t}^{r k}}$ require $K L(K-1)(2 v+$
1) $(2+(K-1)(2 v+1)) \approx \mathcal{O}\left(K^{3} L\right)$ operations. Consequently, the complexity of algorithm 1 is in:

$$
\mathcal{C}_{J A} \approx \mathcal{O}\left(T_{c, \max }\left[K^{3} L+(K N)^{1.5}(K+L)^{2} \ln (1 / \delta)\right]\right)
$$

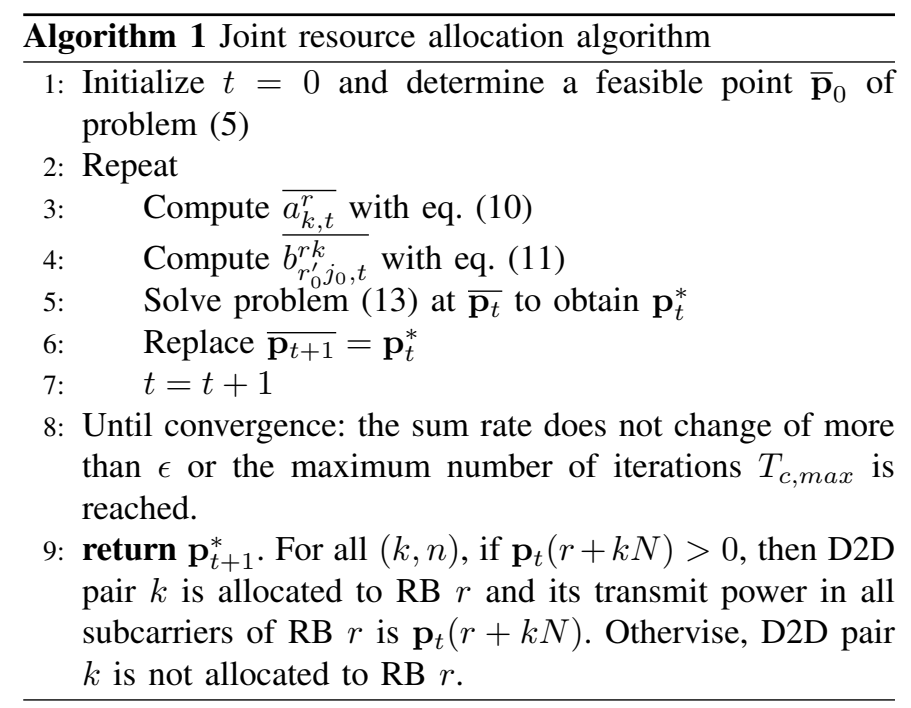

\section{Simulation RESUlTS AND ANALYSis}

\section{A. Simulation assumptions}

Monte-Carlo simulations are conducted with the parameters given in Table I. The bandwidth $B$ is equal to $1.4 \mathrm{MHz}$, corresponding in LTE to a FFT size of 128 and $N=6 \mathrm{RB}$. The carrier frequency is $f_{c}=2.6 \mathrm{GHz}$. We consider $C=6$ cellular users and $K=6$ D2D pairs. The D2D transmitters locations follow a uniform distribution in the cell and their receiver is uniformly located around the transmitter within 50 $\mathrm{m}$.

TABLE I

SIMULATION PARAMETERS

\begin{tabular}{|l|c|}
\hline Cell radius & $500 \mathrm{~m}$ \\
\hline Maximum distance in D2D pair & $50 \mathrm{~m}$ \\
\hline$P_{\max }$ & $21 \mathrm{dBm}$ \\
\hline Noise power spectral density & $-174 \mathrm{dBm} / \mathrm{Hz}$ \\
\hline Path loss model to BS $(d$ in $\mathrm{km})$ & $128.1+37.6 \log _{10}(d)$ \\
\hline Path loss model to devices $(d$ in km $)$ & $140+36.8 \log _{10}(d)$ \\
\hline Shadowing standard deviation, BS & $9 \mathrm{~dB}$ \\
\hline Shadowing standard deviation, devices & $4 \mathrm{~dB}$ \\
\hline Fading from devices & Indoor Channel-B model \\
\hline Fading from cellular & Pedestrian-B model \\
\hline
\end{tabular}

Resource allocation for cellular transmitters is performed as follows: the same number of $\mathrm{RB}$ are allocated to each cellular user randomly. If the whole bandwidth is not occupied, then some cellular users get additional RB. Then power is allocated so as to achieve a SINR of $10 \mathrm{~dB}$ per RB, where the interference is supposed equal to $I_{0}$ and the geometric mean of the channel gain is considered in each RB. Resource allocation is not optimized for cellular users since the objective is here to optimize that of D2D pairs, taking into account an inevitable interference coming from cellular users. 
The proposed algorithm is compared with three algorithms: first, an algorithm called Frequency Division Multiple Access (FDMA) where D2D pairs cannot be multiplexed. RB are allocated to D2D pairs similarly to the previously-described procedure for cellular users. The transmit power per D2D is then equal to the minimum between $P_{\max }$ and the maximum transmit power leading to an interference equal to $I_{0}$.

The second algorithm, called Graph-Based Algorithm (GBA), performs RB allocation on the whole band with graphcoloring: D2D pairs $k$ and $j$ are forbidden to transmit in the same RB if their distance is lower than a given threshold, chosen equal to $125 \mathrm{~m}$ in the simulations. Then graphcoloring is performed with a modified version of greedy Degree SATURation (DSATUR) [13] algorithm, where at each step, when a new edge is added in a color, instead of choosing the first allowed color, the edge chooses the color that has the smallest cardinal. Consequently, graph-coloring eventually provides colors with almost the same cardinality, thus generating lower intra-group interference levels. The total number of RB is divided by the number of groups. Adjacent $\mathrm{RB}$ form a set of RB allocated to one group of user. If the number of RB $N$ divided by the number of groups $S_{g}$ is not an integer, then to occupy the whole bandwidth, we use the following method: Let $s^{\prime}=N \bmod \left(S_{g}\right)$. The first $s^{\prime}$ groups get $\left\lfloor\frac{N}{S_{g}}\right\rfloor+1 \mathrm{RB}$, and the remaining $S_{g}-s^{\prime}$ groups get $\left|\frac{N}{S_{g}}\right|$ RB. This RB allocation has a complexity in $\mathcal{O}\left(N K^{2}\right)$. Finally, the high SINR power control algorithm from [5] is used.

The third algorithm separates RB from power allocation and was presented in [3]. RB are firstly allocated with the infinity norm criterion to guarantee that all D2D pairs that are allocated on a given RB could achieve the target SINR per subcarrier of $\gamma=10 \mathrm{~dB}$, if the BS interference constraint was not taken into account. This provides a subset of D2D pairs that do not highly interfere each others and that can be multiplexed. Then, power is allocated under high SINR assumption. This distributed algorithm is referred to as 'DA'.

\section{B. Convergence study}

Firstly, the convergence of the proposed algorithm is numerically studied. With a convergence criterion $\epsilon=1 \%$, in average, 7 iterations are required for Alg. 1 to converge, whatever the multi-carrier modulation. This result does only slightly depend on the value of $I_{0}$.

\section{Average data rate}

Secondly, the average data rate is represented on Fig. 1 with all algorithms for FBMC and OFDM, and on Fig. 2 with CA and all multi-carrier modulations. Fig. 1 shows that the average data rate per D2D pair is higher with CA than with all other tested algorithms. CA provides a data rate increase of $8.5 \%$ compared to DA, of $36 \%$ compared to GBA and of $292 \%$ compared to FDMA. Moreover, Fig. 2 shows the average data rate achieved with CA with four different multi-carrier modulations, and with the theoretical Perfect Synchronization case. The rate decreases is up to $11 \%$ with asynchronous OFDM compared to PS. It is limited to $0.9 \%$ with FFT-FBMC and to $1 \%$ with FBMC.

Fig. 3 represents the Cumulative Distribution Function (CDF) of the data rate per D2D pair with FBMC and OFDM.

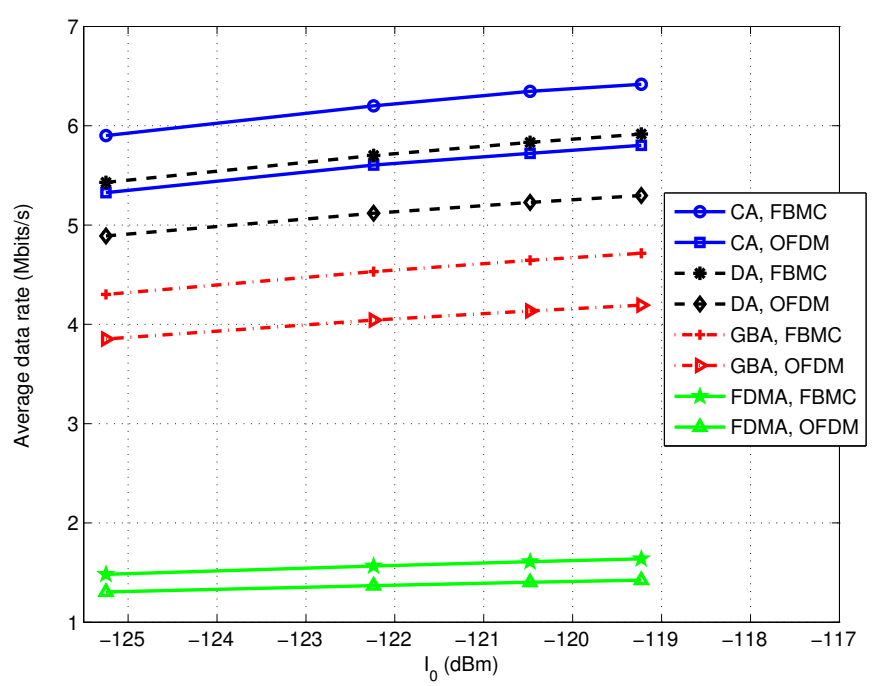

Fig. 1. Average rate per D2D pair vs. $I_{0}$, FBMC and OFDM

We can notice that CA is less fair than the other algorithms, and that the difference in average data rate is due to some D2D pairs that obtain very large data rates when CA is used. It should be noted that we here only consider one Transmit Time Interval (TTI). If the proposed algorithm was used in conjunction with a scheduling algorithm over a large number of TTI, fairness could be obtained by adding a fairness constraint in the optimization problem. For instance, proportional fairness could be achieved by maximizing the weighted sum rate, with D2D pairs' weights inversely proportional to their cumulative rate.

\section{Average transmit power}

Finally, the average transmit power per D2D transmitter is depicted on Fig. 4, and the CDF of the transmit power in two subcases is shown on Fig. 5. With CA, many D2D transmitters are inactive, but some of them use large transmit powers. This is consistent with the data rate results: CA provides more $\mathrm{RB}$ to a subset of $\mathrm{D} 2 \mathrm{D}$ pairs, that consequently transmit with higher power values. Moreover, using multicarrier modulations with low ICI spread such as FFT-FBMC and FBMC is not only efficient with respect to the achieved data rate, but also with respect to the power budget, since interference is then easier to manage and does not need to be compensated by higher transmit powers.

\section{CONCLusions}

This paper has proposed a joint multiplexing, RB and power allocation algorithm for asynchronous D2D underlay communications. It achieves higher D2D sum rates than three different algorithms. Moreover, in asynchronous transmissions, FFT-FBMC and FBMC are almost as efficient as if all signals were received synchronously. These results emphasize the fact that choosing multi-carrier modulations that are well-localized in the frequency domain is an important feature for future Beyond 5G systems, where the synchronization constraint may be relaxed. 


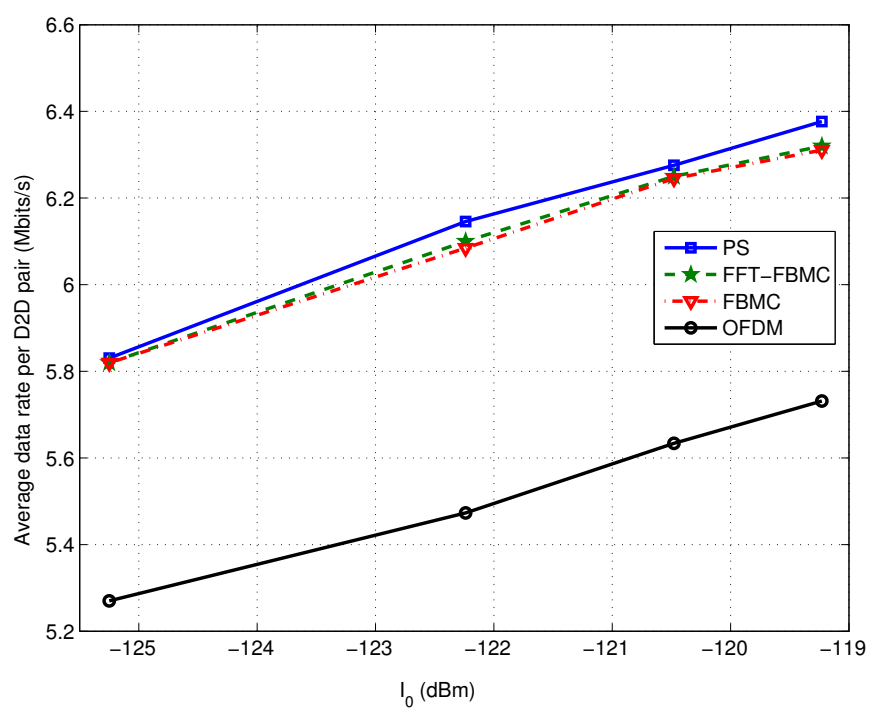

Fig. 2. Average rate per D2D pair vs. $I_{0}, \mathrm{CA}$

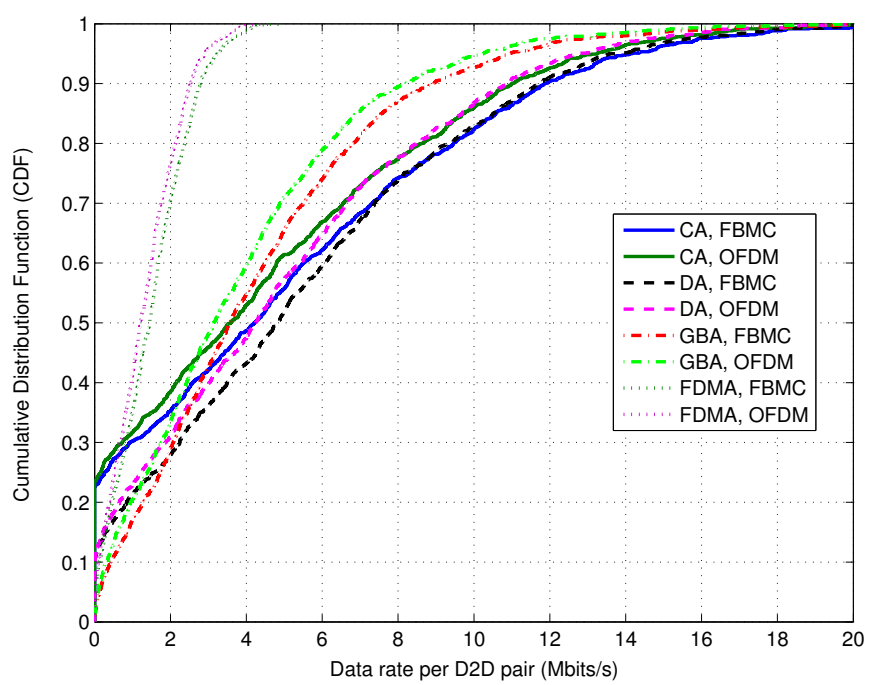

Fig. 3. CDF of data rate per D2D pair when $I_{0}=5 n_{0}$

\section{ACKNOWLEDGEMENT}

This work was partially funded through French National Research Agency (ANR) project ACCENT5 with grant agreement code: ANR-14-CE28-0026-02.

\section{REFERENCES}

[1] A. Asadi, Q. Wang, and V. Mancuso, "A Survey on Device-to-Device Communication in Cellular Networks," IEEE Communications Surveys and Tutorials, vol. 16, pp. 1801 - 1819, 4rth quarter 2016.

[2] W. Zhao and S. Wang, "Resource Sharing Scheme for Device-to-Device Communication Underlaying Cellular Networks," IEEE Transactions on Communications, vol. 63, pp. 4838-4848, Dec. 2015.

[3] B. Ozbek, M. Pischella, and D. Le Ruyet, "Dynamic shared spectrum allocation for underlaying device-to-device communications," IEEE Wireless Communications, vol. 24, no. 5, pp. 88-93, October 2017.

[4] R. Zakaria and D. Le Ruyet, "A novel filter-bank multicarrier scheme to mitigate the intrinsic interference: Application to MIMO systems ," IEEE Trans. on Wireless Communication, vol. 11, no. 3, pp. 1112-1123, March 2012

[5] M. Pischella, R. Zakaria, and D. Le Ruyet, "Resource Block level power allocation in asynchronous multi-carrier D2D communications ," IEEE Communication Letters, vol. 21, no. 4, pp. 813-816, Apr. 2017.

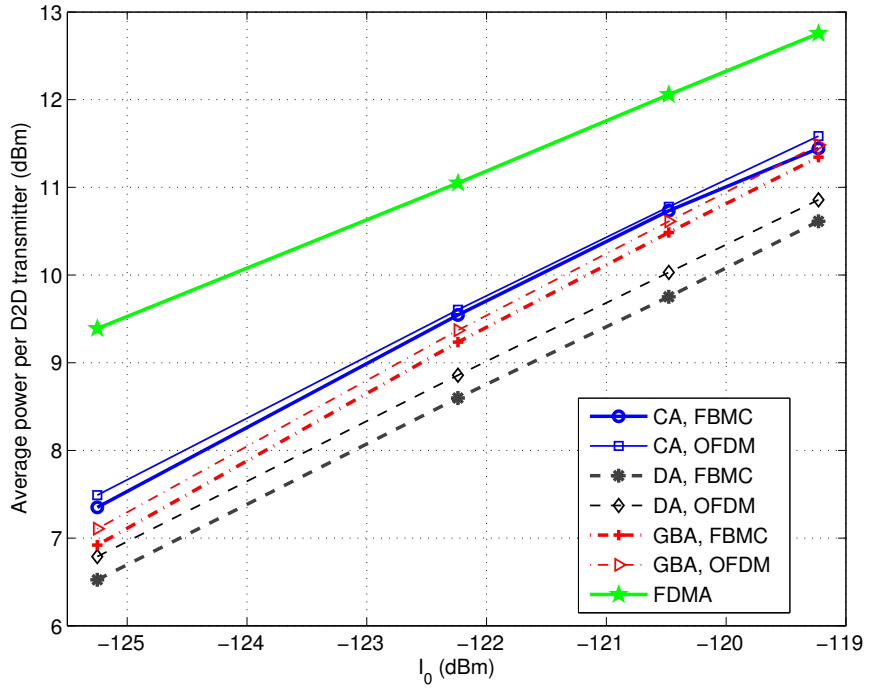

Fig. 4. Average transmit power per D2D transmitter vs. $I_{0}$, FBMC and OFDM

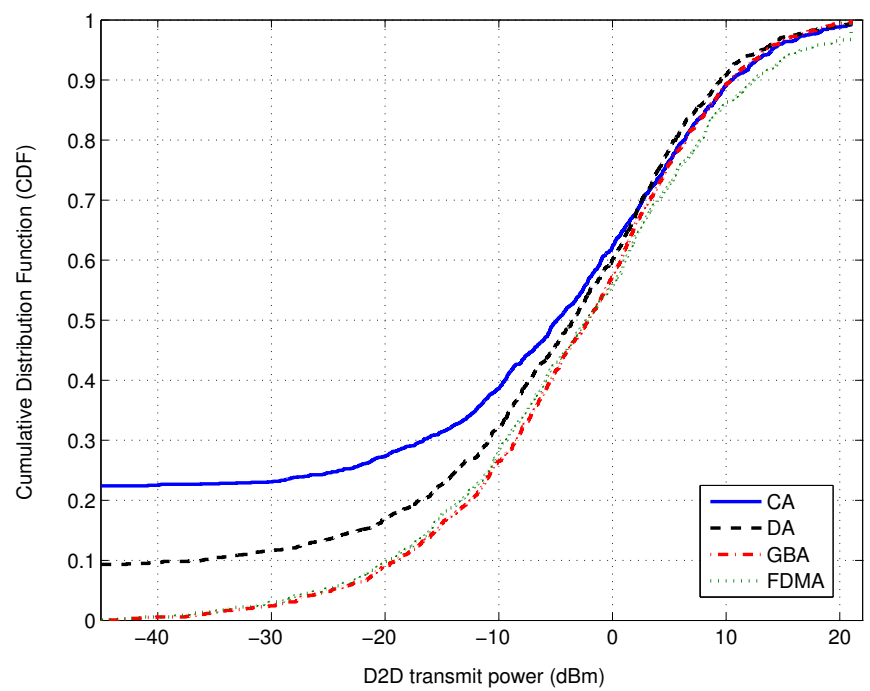

Fig. 5. CDF of transmit power when $I_{0}=5 n_{0}$, with FBMC, low bandwidth scenario

[6] P. Mach, Z. Becvar, and T. Vanek, "In-band Device-to-Device communication in OFDMA cellular networks: a survey and challenges," IEEE Comm. Surveys and Tutorials, vol. 17, no. 4, pp. 1885-1922, Oct. 2015.

[7] T.D. Hoang, L.B. Le, and T. Le-Ngoc, "Joint subchannel and power allocation for D2D communications in cellular networks," in Proc. of WCNC 2014, Istanbul, Turkey, April 2014.

[8] T.D. Hoang, L.B. Le, and T. Le-Ngoc, "Power allocation for D2D communications under proportional fairness," in Proc. of Globecom 2014, Austin, TX, USA, Dec. 2014.

[9] M. G. Bellanger, "Specification and Design of a Prototype Filter for Filter Bank based Multicarrier Transmission," in Proc. of ICASSP 2001, Salt Lake City, UT, USA, May 2001.

[10] Barry R. Marks and Gordon P. Wright, "A general inner approximation algorithm for nonconvex mathematical programs," Operations Research, vol. 26, pp. 681-683, July-Aug. 1978.

[11] M. Grant and S. Boyd, "CVX: Matlab software for disciplined convex programming, version 2.1," in http://cvxr.com/cvx/, Oct 2016.

[12] A. Nemirovski, "Interior point polynomial time methods in convex programming: lecture notes," in Georgia institute of technology, Spring Semester 2004

[13] D. Brélaz, "New methods to color the vertices of a graph," Communications of the ACM, vol. 22, no. 4, pp. 251-256, Apr. 1979. 